\title{
CELL AVERAGING CFAR DETECTOR WITH SCALE FACTOR CORRECTION THROUGH THE METHOD OF MOMENTS FOR THE LOG-NORMAL DISTRIBUTION
}

\author{
DETECTOR CFAR DE PROMEDIACIÓN CON CORRECCIÓN DEL \\ FACTOR DE AJUSTE A TRAVÉS DEL MÉTODO DE LOS MOMENTOS \\ PARA LA DISTRIBUCIÓN LOG-NORMAL
}

José Raúl Machado Fernández*

Jesús C. Bacallao Vidal*

Fecha de recepción: 1 de noviembre de 2016

Fecha de revisión: 28 de abril de 2017

Fecha de aprobación: 31 de mayo de 2017

Cómo citar: J. R. Machado Fernández y J. C. Bacallao Vidal, “Cell Averaging CFAR Detector with Scale Factor Correction through the Method of Moments for the Log-Normal Distribution," Ciencia e Ingeniería Neogranadina, vol. 28, no. 1, pp. 27-44. DOI: https://doi.org/10.18359/rcin.2408

\section{ABSTRACT}

The new LN-MoM-CA-CFAR detector is introduced, exhibiting a reduced deviation of the operational false alarm probability from the value conceived in the design. The solution solves a fundamental problem of CFAR processors that has been ignored in most proposals. Indeed, most of the previously proposed schemes deal with sudden changes in the clutter level, whereas the new solution has an improved performance against slow statistical changes that occur in the background signal. It has been proven that these slow changes have a remarkable influence on the selection of the CFAR adjustment

\footnotetext{
* Ingeniero en Telecomunicaciones y Electrónica, máster en Telecomunicaciones y Electrónica y doctorante en Ciencias Técnicas. Profesor e investigador, Grupo de Investigación de Radares, Departamento de Telecomunicaciones y Telemática, de la Facultad de Eléctrica, Instituto Superior Politécnico José Antonio Echeverría (ISPJAE-CUJAE), La Habana, Cuba. Correo electrónico: josemf@electrica.cujae.edu.cu. ORCID: http://orcid. org/0000-0002-9185-5033

** Ingeniero Eléctrico y doctor en Ciencias Técnicas. Profesor titular e investigador, 2do. jefe del Grupo de Investigación de Radares, Departamento de Telecomunicaciones y Telemática de la Facultad de Eléctrica del Instituto Superior Politécnico José Antonio Echeverría (ISPJAE-CUJAE), La Habana, Cuba. Correo electrónico: bacallao@electrica.cujae.edu.cu. ORCID: http://orcid.org/0000-0003-4224-7565
} 
factor, and consequently in maintaining the false alarm probability. The authors took advantage of the high precision achieved by the MoM (Method of Moments) in the estimation of the Log-Normal (LN) shape parameter, and the wide application of this distribution to radar clutter modeling, to create an architecture that offers precise results and it's computationally inexpensive at the same time. After an intensive processing, involving 100 million Log-Normal samples, a scheme, which operates with excellent stability reaching a deviation of only $0,2884 \%$ for the probability of false alarm of 0,01 , was created, improving the classical CA-CFAR detector through the continuous correction of its scale factor.

Keywords: Method of moments, CFAR detectors, false alarm probability, Log-Normal distribution, radar clutter.

\section{RESUMEN}

Se presenta el nuevo detector LN-MoM-CA-CFAR que tiene una desviación reducida en la tasa de probabilidad de falsa alarma operacional con respecto al valor concebido de diseño. La solución corrige un problema fundamental de los procesadores CFAR que ha sido ignorado en múltiples desarrollos. En efecto, la mayoría de los esquemas previamente propuestos tratan con los cambios bruscos del nivel del clutter mientras que la presente solución corrige los cambios lentos estadísticos de la señal de fondo. Se ha demostrado que estos tienen una influencia marcada en la selección del factor de ajuste multiplicativo CFAR, y consecuentemente en el mantenimiento de la probabilidad de falsa alarma. Los autores aprovecharon la alta precisión que se alcanza en la estimación del parámetro de forma Log-Normal con el MoM, y la amplia aplicación de esta distribución en la modelación del clutter, para crear una arquitectura que ofrece resultados precisos y con bajo costo computacional. Luego de un procesamiento intensivo de 100 millones de muestras Log-Normal, se creó un esquema que, mejorando el desempeño del clásico CA-CFAR a través de la corrección continua de su factor de ajuste, opera con una excelente estabilidad alcanzando una desviación de solamente $0,2884 \%$ para la probabilidad de falsa alarma de 0,01.

Palabras clave: método de los momentos, detectores CFAR, probabilidad de falsa alarma, distribución Log-Normal, clutter de radar.

\section{INTRODUCTION}

A radar is a device that emits electromagnetic waves and gathers the resulting echo caused by objectives in the proximity [1]. The mission of the radar is to detect nearby tar- gets of interest and to discard those that do not relate to a particular application. Therefore, some objectives (such as clouds) can be considered as targets for certain applications (meteorology) and as an interfering signal (warfare) for others [2]. 
Echo signals produced at the ground surface, sea surface or weather volumes (clouds, fog, rain) are assumed as interference and called clutter, in most radar applications [3]. The magnitude of the clutter signal cannot be deducted by purely deterministic mechanisms. Hence, its modeling falls in the field of statistics.

The false alarm probability $\left(P_{f}\right)$ is one of the radar fundamental parameters because it defines the frequency of occurrence of type 1 errors. A type 1 error takes place when a clutter signal is mistakenly classified as a target [4].

Although decreasing the $P_{f}$ is beneficial; generally, it has the undesirable effect of disturbing the probability of detection $\left(P_{D}\right)$, which is another essential parameter. To resolve this 'balance' relation, the Neyman-Pearson criterion is applied, which states that a certain level of $P_{f}$ must be guaranteed first as an essential requirement. Only afterward, actions can be taken to improve the $P_{D}$ by modifying other variables. Therefore, radar detectors or processors must have the CFAR (Constant False Alarm Rate) property, because they must ensure a constant $P_{f}$ is maintained the entire period of operation [5]. The idea behind the CFAR detection is to adapt the Neyman-Pearson detector to a signal with a variable mean.

The classical radar detector, known as CACFAR (Cell Averaging CFAR), uses a sliding window with multiple slots that it's passed through the whole coverage area. Thus, each resolution cell has a chance to occupy the central position of the window. The processor evaluates the cell in this position deciding on the presence or absence of a target. The infor- mation in the neighboring cells is used to calculate the average of the clutter, which is then multiplied by a scale or adjustment factor $(T)$, resulting in the establishment of a detection threshold. If the magnitude of the sample in the central slot is greater than the threshold, a target is considered to be present [6].

\section{MOTIVATION AND OBJECTIVE}

While the CA-CFAR guarantees a constant $P_{f}$ for several radar scenarios, the occurrence of multiple close targets, and the appearance of sudden changes in the clutter level, disturb the mechanism of average calculation, provoking deviations from the design $P_{f}[6]$. To prevent malfunctions against these critical situations, multiple alternative detectors have been created, being all variations from the original CA-CFAR [7]-[10].

Nevertheless, most authors ignore the effect of clutter slow statistical changes in the maintaining of the $P_{f}$. Their designs lack an adaptive correction of the multiplicative factor and focus exclusively on changing the mechanism for calculating the average.

In contradiction, a lot of papers have verified the need to modify the shape parameter of the distributions used as clutter models for achieving a quality fit with empirical data [11][14]. The variation in the distribution needs to be translated into a correction of the CFAR factor for guaranteeing the stability of the system. Indeed, in [15] it was demonstrated, by performing simulations with computer-generated data, that a processor with a constant $T$ value was unable to maintain its design $P_{f}$ when it was tested with clutter having a shape parameter that changed over time. 
To solve the above-described problem, the authors aimed at creating an improved CFAR detector, able to correct its $T$ factor according to the clutter statistical variations. For this purpose, they selected the Log-Normal (LN) distribution as a model for clutter and the CA-CFAR detector as a reference implementation where the improved solution will be introduced. The Method of Moments (MoM) was the mathematical tool used in the estimation of the LN shape parameter. Therefore, the new scheme was baptized as the LN-MoM-CA-CFAR processor.

\section{MATERIALS AND METHODS}

This section presents the structure of the LN-MoM-CA-CFAR scheme and explains its internal blocks. Particular attention is given to the description of the LN statistical mo- del, the derivation of MoM expressions and the selection of the range of possible values for the shape parameter. Subsequently, the experiments required to test the scheme in MATLAB are described.

\subsection{The LN-MOM-CA-CFAR scheme}

The LN-MoM-CA-CFAR scheme was constructed by adding four blocks to the classical CA-CFAR as shown in Fig. 1. Samples of radar readings are fed simultaneously to the input of the CA-CFAR and the block for calculating the average. Afterwards, the variance is calculated using, as it was also done for the mean, 3000 statistically independent samples. This amount of samples was selected after consulting procedures followed by other authors [16]-[18].

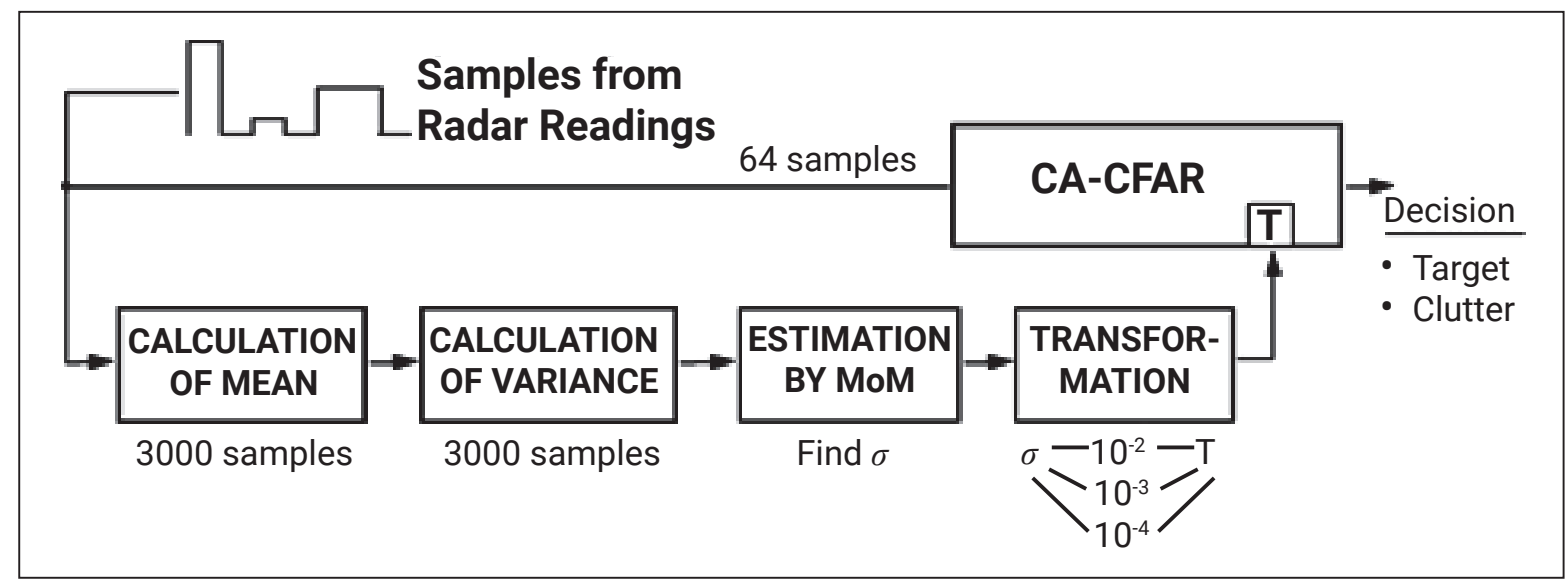

Fig. 1 Structure of the LN-MoM-CA-CFAR scheme Source: The authors. 
The CA-CFAR detector uses only the last 65 cells of the 3000 fed to the "Calculation of Mean" block. The cell at the center of the last 65 is interpreted as the CUT (Cell Under Test), while the rest are placed in the sliding window. Then the average of the cells in the window is multiplied by the scale factor $(T)$, which results in the detection threshold. If the magnitude of the CUT is superior to the threshold, then this cell is classified as a target.

The main advantage of using the $\mathrm{LN}$ distribution as preferential clutter model is the straightforward derivation of the parameters by the Method of Moments. Most distributions related to radar clutter (such as Weibull or $\mathrm{K}$ ) allow a quick estimation by the MoM, but the accuracy of the result is limited. However, the MoM concurs with the expression of ML (Maximum Likelihood) for the LN distribution, thanks to its close relationship with the Normal or Gaussian distribution. So, the estimation of the LN parameters can be made in a quickly and accurately way [19].

In Fig. 1, the "Calculation of Mean" block estimated the mean of the natural logarithm of the samples. The same goes for the block "Calculation of variance". These are the requirements for LN MoM formula which is given in Equations (4) and (5).

Once estimated LN shape parameter $(\sigma)$, it is necessary to translate it into a viable correction of the $T$ value. As the selection of $T$ depends on the wanted false alarm rate, the authors selected three classical $P_{f}$ values to design the solution: $P_{f}=10^{-2}, P_{f}=10^{-3}$ and $P_{f}=$ $10^{-4}$. These figures are a common choice in radar related investigations and were previously used in [20], [21]. The designer may choose any of these $P_{f}$ according to its requirements.

\subsection{The Log-Normal statistical} distribution and the mom

The LN statistical distribution has had an extensive application in radars issues. It is widely used for both sea [12], [13], [16], [22] and ground clutter [23]-[25]. Even if the model prevails sometimes for a complete set of low grazing angle measurements [16], [26], its best fit generally appears for sub-sets with $\mathrm{HH}$ polarization [27], when measuring the spatial data spatial distribution [25], and for cells containing big targets reflections [16], [28].

In [29], [30] the following expression was used for the LN PDF (Probability Density Function):

$$
f(x \mid \mu, \sigma)=\frac{1}{\sigma x \sqrt{2 \pi}} \exp \left[-\frac{1}{2}\left(\frac{\ln (x)-\mu}{\sigma}\right)^{2}\right]
$$

Where $\mu$ and $\sigma$ are the scale and shape parameters respectively, and $x$ represents the clutter amplitude measurements. The CDF (Cumulative Density Function) [31] and the expression for the algebraic moments [31] are given below:

$$
\begin{gathered}
F(x)=\frac{1}{2}\left(1+\operatorname{erf}\left(\frac{\ln x-\mu}{\sigma \sqrt{2}}\right)\right) \\
M_{n}=\exp \left(n \mu+\frac{(n \sigma)^{2}}{2}\right)
\end{gathered}
$$


Where $\operatorname{erf}($.$) is the error function, \mu$ is the logarithmic average and $\sigma^{2}$ is the variance of $\ln x^{2}$.

The MoM derivation for the LN case, addressed in [19], allows estimating the parameters of the distribution with the following expressions:

$$
\begin{gathered}
\mu=\frac{1}{N} \sum_{i=1}^{n} \ln \left(x_{i}\right) \\
\sigma^{2}=\frac{1}{N-1}\left\{\sum_{i=1}^{N} \ln \left(x_{i}\right)^{2}-\mu^{2}\right\}
\end{gathered}
$$

Where $N$ is the total amount of samples, $i$ is the number of the current sample, which goes from 1 to $N$, and $x_{i}$ is the $i-t h$ sample of the set.

While the LN distribution allows any configuration of its parameters from a mathematical point of view, not all combinations reflect real situations. After a review of the related literature, the authors decided that the $\sigma$ interval between 0,025 and 1,25 covers most of the operating conditions [24], [27], [32], [33].

\subsection{Design of the experiments}

The mathematical foundations of the proposed scheme were given previously, together with the leading precedents of the literature. The following experiments were carried out by the authors to build and test the LN-MoMCA-CFAR processor.

\subsubsection{Building the transformation block}

Firstly, the authors implemented the scheme from Fig. 1 in MATLAB. The main block of the design is "Transformation" which cannot be obtained by any of the previously described methods. Therefore, the authors dedicated the first experiment to obtain the relationship between the $\mathrm{LN} \sigma$ and the CACFAR $T$, for a CA-CFAR having 64 cells in the reference window.

The methodology followed was similar to that described in [20], [21]. An amount of 40 $\sigma$ values, uniformly distributed in the range between 0,025 and 1,25, were chosen and a group of one million $\mathrm{LN}$ samples was generated for each $\sigma$ value. Fig. 2 shows the structure of the created set of samples, which will be referred from now on as Set $A$.

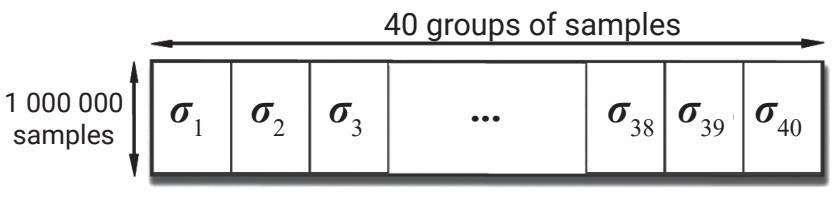

Fig. 2 Structure of the Set A that contains 40 million Log-Normal Samples

Source: The authors.

After generating the data, the first group from Set $A$ was processed by a CA-CFAR operating with a low $T$ value (for example $T=0,90$ ). Then, the $P_{f}$ was measured. As the initial $T$ value was low, the resulting $P_{f}$ was quite high (for example 0,1 ). At this point, one iteration was completed.

In the next iteration, $T$ was raised thereby producing a lower $P_{f}$. Many others iterations were executed until the exact values of $T$ for 
were found for $P_{f}=10^{-2}, P_{f}=10^{-3}$ and $P_{f}=10^{-4}$ with a $1 \%$ deviation. At this point, the process ended for the first group of samples.

A similar experiment was performed on the remaining 39 groups, yielding a total of $120 \mathrm{~T}$ values as a result of the test. With these values, a curve fitting procedure was carried out obtaining three mathematical expressions through which the optimal $T s$ can be easily obtained for any $\sigma$, and any of the three addressed $P_{f} \mathrm{~s}$. These three expressions were placed in the "Transformation" block from Fig. 1.

\subsubsection{Mistakes committed by the method of moments}

It was necessary to quantify the error committed by the "Estimation by MoM" block from Fig. 1 to derive the overall accuracy of the scheme. For this purpose, the authors prepared a Set B composed of 20000 groups of 3 000 samples each; see Fig. 3. As it was done for Set A, the $\sigma$ of each group was increased uniformly in the established interval.

20 groups of samples

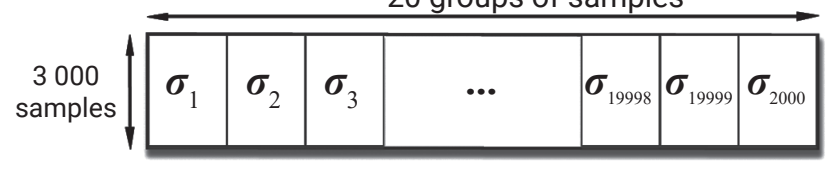

Fig. 3 Structure of Set B that contains 60 million Log-Normal Samples

Source: The authors.

Each group from Set B was processed with the MoM according to expressions (4) and (5). The incurred error was quantified and arranged into histograms for its better characterization.

\subsubsection{Influence of the MoM error over} the selection of $T$

The influence of the MoM error in the CACFAR multiplier $(T)$ selection was studied next. Set B itself was used in the trials.

The test was divided into two parts. In the first one, samples were evaluated with a CACFAR with a priori knowledge of the $\sigma$ of each group. In the second, the procedure was repeated using the MoM $\sigma$ estimation instead of the original $\sigma$. The difference between the two obtained $T$ revealed the committed error.

\subsubsection{Influence of $T$ selection on the false alarm rate}

After knowing the mistake made in $T$ selection, a final experiment was conducted to find the deviation of the operational $P_{f}$ from the design value. As it was expected, a higher error in the selection of $T$ resulted in an increased deviation of the $P_{f}$.

However, the error does not spread with an easy to deduce mechanism, as the decision taken by the detector is always drastic. Regardless of the margin by which the decision was made, the result remains the same as the detector always chooses between 1 and 0 , (i.e between target and clutter). There are no intermediate levels.

For this test, a variation of Set B, named Set $C$, was used. Set $C$ originates from separating the 20000 groups from Set $A$ into 20 sections of 1000 groups each. The purpose of these sections is to lighten the processing load, and they didn't introduce any changes in the data. 
Each section from Set $C$ was processed with a CA-CFAR whose $T$ was defined according to estimates made by the MoM for each of the considered $P_{f}$ s. The difference between the expected and the achieved $P_{f}$ revealed the deviation introduced by the MoM and the global precision with which operates the LN-MoM-CA-CFAR solution.

\section{RESULTS AND DISCUSSION}

This section will first show how the "Transformation" block from Fig. 1 was constructed. The construction of the block is, in fact, the most significant contribution of the research as it was the main obstacle in the creation of the LN-MoM-CA-CFAR detector. Secondly, the section will provide evidence of the proper functioning of the new scheme, describing the error introduced by its components and the reduced deviation that it achieves for the operational $P_{f}$.

\subsection{Influence of $\mathrm{T}$ selection on the false alarm rate}

By performing Monte Carlo simulations with the 40 millions of samples contained in Set A, a total of $120 T$ values were obtained for the three addressed $P_{f}$ s. Fig. 4 plots the $T$ values, revealing the remarkable influence of $\sigma$ over $T$ in the $\sigma>0,8$ region.

The cause of the observed tendency lies in the appearance of heavy tails for the higher figures of $\sigma$. A heavy tail produces sporadic high magnitude samples that disturb the most the lower $P_{f} \mathrm{~s}$.

To build the "Transformation" block, mathematical expressions that provide $T$ values for any occurrence of $\sigma$ in the $0,025-1$, 25 region are required. These expressions will avoid the storage of the values plotted in Fig. 4 and will also provide a soft approximation to any $\sigma$ within the interval.

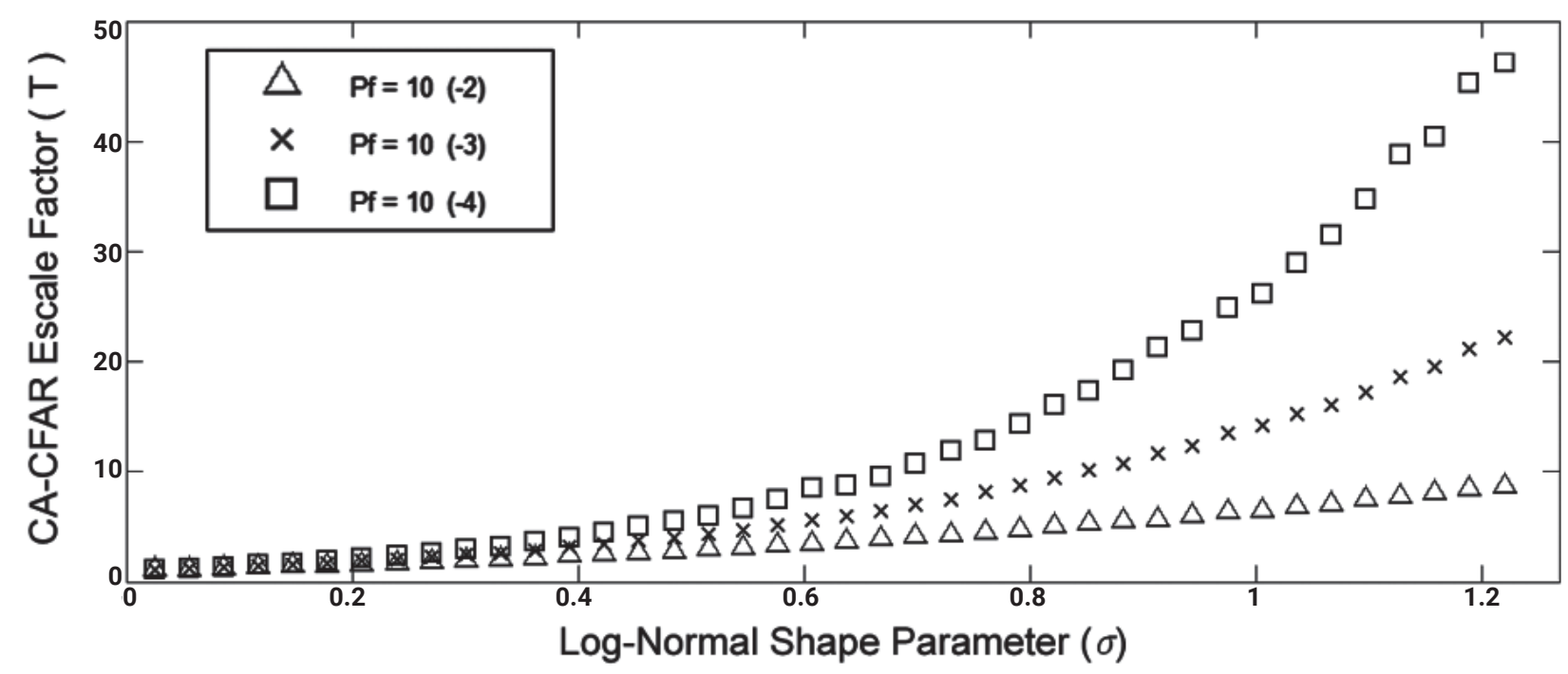

Fig. 4 Optimal values for multiple statistical conditions of Log-Normal distributed clutter Source: The authors. 
After trying different alternatives, the authors concluded that the polynomial fits displayed the best resemblance with the data. Fig. 5 shows, the polynomials fit of first, second and third order for the $P_{f}=10^{-4} \mathrm{case}$. The linear approximation was obviously inappro- priate since it exhibited no resemblance with the data. The quadratic fit was a little closer, but there were several areas where the departure was significant. Instead, the cubic polynomial followed the behavior of the data very closely.
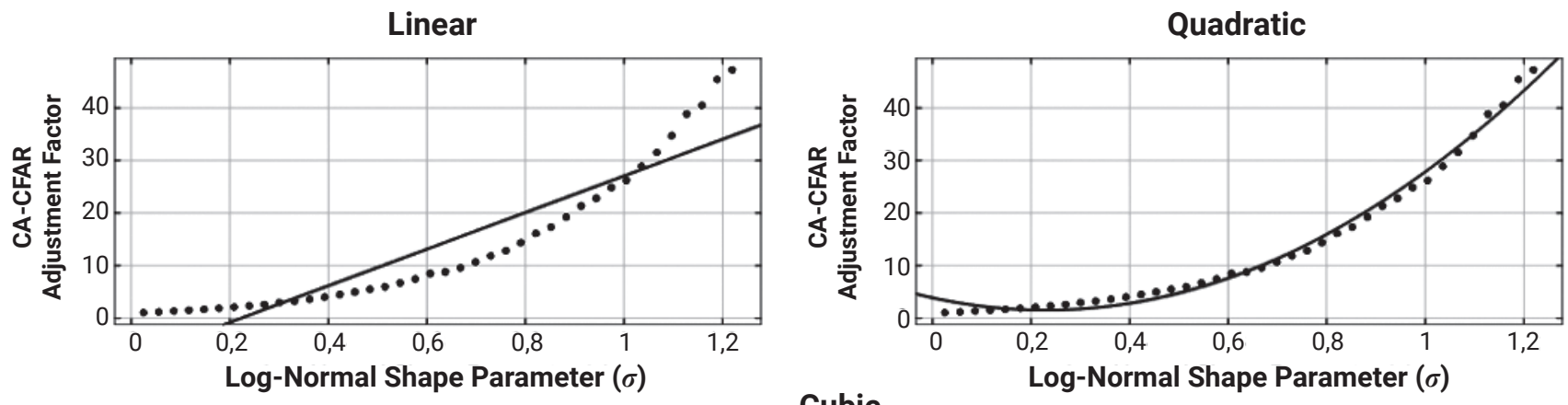

Cubic

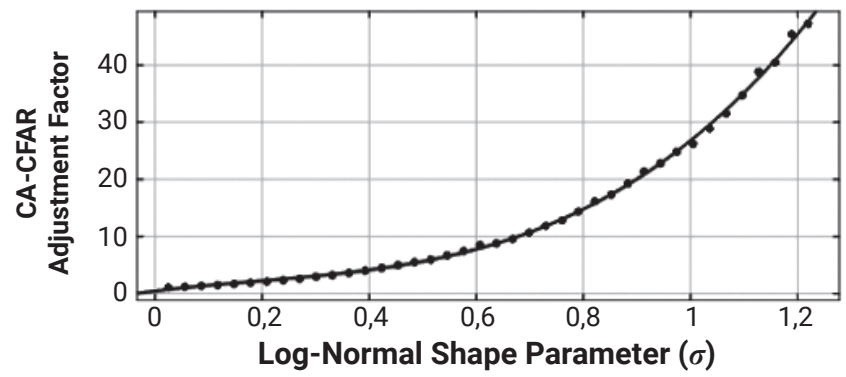

Fig. 5 Low order polynomial fits for data from Fig. 4 corresponding to $P_{f}=10^{-4}$

Source: The authors.

In short, fits from second to eighth order were considered, being the incurred mistake made showed in Table 1 . Note that a constant improvement in accuracy is achieved up until the fourth order, where the RMSE (Root Mean Square Error) and SSE (Sum of Squared Error) reach a saturation point.

Table 1. Results of the polynomial fit

\begin{tabular}{|l|l|l|l|l|l|l|}
\hline $\begin{array}{c}\text { Polynomial } \\
\text { Degree }\end{array}$ & $\begin{array}{c}\text { RMS for } \\
\mathbf{P}_{\mathbf{f}}=\mathbf{1 0}^{-\mathbf{2}}\end{array}$ & $\begin{array}{c}\text { SSE for } \\
\mathbf{P}_{\mathbf{f}}=\mathbf{1 0}^{-\mathbf{2}}\end{array}$ & $\begin{array}{c}\text { RMS for } \\
\mathbf{P}_{\mathbf{f}}=\mathbf{1 0}^{-\mathbf{3}}\end{array}$ & $\begin{array}{c}\text { SSE for } \\
\mathbf{P}_{\mathbf{f}}=\mathbf{1 0}^{-\mathbf{3}}\end{array}$ & $\begin{array}{c}\text { RMS for } \\
\mathbf{P}_{\mathbf{f}}=\mathbf{1 0}^{-\mathbf{4}}\end{array}$ & \multicolumn{1}{|c|}{$\begin{array}{c}\text { SSE for } \\
\mathbf{P}_{\mathbf{f}}=\mathbf{1 0}^{-\mathbf{4}}\end{array}$} \\
\hline 2 & 0,5783 & 2,755 & 0,8484 & 4,0392 & 1,286 & 6,12 \\
\hline 3 & 0,1826 & 2,6679 & 0,2679 & 3,9124 & 0,4058 & 5,928 \\
\hline 4 & 0,1748 & 2,2741 & 0,2552 & 3,3364 & 0,3872 & 5,055 \\
\hline
\end{tabular}


CELL AVERAGING CFAR DETECTOR WITH SCALE FACTOR CORRECTION THROUGH THE METHOD OF MOMENTS

FOR THE LOG-NORMAL DISTRIBUTION

\begin{tabular}{|l|l|l|l|l|l|l|}
\hline $\begin{array}{c}\text { Polynomial } \\
\text { Degree }\end{array}$ & $\begin{array}{c}\text { RMS for } \\
\mathbf{P}_{\mathbf{f}}=\mathbf{1 0}^{-\mathbf{2}}\end{array}$ & $\begin{array}{c}\text { SSE for } \\
\mathbf{P}_{\mathbf{f}}=\mathbf{1 0}^{-\mathbf{2}}\end{array}$ & $\begin{array}{c}\text { RMS for } \\
\mathbf{P}_{\mathbf{f}}=\mathbf{1 0}^{-\mathbf{3}}\end{array}$ & $\begin{array}{c}\text { SSE for } \\
\mathbf{P}_{\mathbf{f}}=\mathbf{1 0}^{-\mathbf{3}}\end{array}$ & $\begin{array}{c}\text { RMS for } \\
\mathbf{P}_{\mathbf{f}}=\mathbf{1 0}^{-\mathbf{4}}\end{array}$ & $\begin{array}{c}\text { SSE for } \\
\mathbf{P}_{\mathbf{f}}=\mathbf{1 0}^{-\mathbf{4}}\end{array}$ \\
\hline 5 & 0,1733 & 2,2703 & 0,2547 & 3,3307 & 0,3852 & 5,046 \\
\hline 6 & 0,1751 & 2,2545 & 0,2570 & 3,3060 & 0,3896 & 5,009 \\
\hline 7 & 0,1743 & 2,152 & 0,2558 & 3,1623 & 0,3869 & 4,791 \\
\hline 8 & 0,1709 & 2,0595 & 0,2497 & 3,0205 & 0,3781 & 4,576 \\
\hline
\end{tabular}

Source: The authors.

Consequently, the authors selected the fourth-order polynomial fit for the expressions that composed the "Transformation" block from Fig. 1. Therefore, the CA-CFAR optimal $T s$ can be obtained through (6), (7) and (8), corresponding to the $P_{f} s$ of $10^{-4}, 10^{-3}$ and $10^{-2}$ respectively.

$T=13,91 \sigma^{4}-0,624 \sigma^{3}+8,729 \sigma^{2}+3,608 \sigma+0,994$

$T=2,56 \sigma^{4}+2,407 \sigma^{3}+5,247 \sigma^{2}+2,839 \sigma+1,018$

$T=-0,1728 \sigma^{4}+0,8586 \sigma^{3}+2,558 \sigma^{2}+2,236 \sigma+1,007$

Alternatively, it was found that the fit for rational expressions, such as (9), also achieved a good approximation with the data.

$$
f(x)=\frac{\left(p_{1} x+p_{2}\right)}{\left(x^{2}+q_{1} x+q_{2}\right)}
$$

However, the RMSE of the rational fit was slightly higher compared to the fourth order polynomial RMSE. Thus, the latter was preferred.

\subsection{Error in the LN shape parameter estimation}

When the $\sigma$ of the clutter is known a prio$\mathrm{ri}$, the optimal $T$ can be chosen to operate with the desired $P_{f}$ using expressions (6), (7) and (8). However, in a real operating environment, $\sigma$ is unknown, and the proposed scheme performs its estimation through the MoM. The resulting estimate will be slightly deviate from the real value because a finite set of samples was used in the process.

Fig. 6 shows the error committed by the LN-MoM-CA-CFAR in the estimation of $\sigma$ after evaluating Set A for $P_{f}=10^{-4}$. The results are appropriate since they are equally distributed in positive and negative magnitudes. This is easily visible in Fig. 7 that plots a histogram of the errors.

The average error was 0.006566 , and the maximum error was 0.048 . These are excellent results because they represent mistakes of only $0.5 \%$ and $5 \%$ respectively. 


\subsection{Deviation of the adjustment factor}

The error incurred in the estimation of $\sigma$ affects the choice of the adjustment factor $(T)$. To determine the deviation of the $T$ estimates, experiments were conducted with Set B. Fig. 8 shows the deviation of the absolute error in the adjustment factor for $P_{f}=10^{-4}$. Fig. 9 does the same with the percentage of the relative error.
The mean absolute error in the selection of $T$ for $P_{f}=10^{-4}$ was 0.3946 , and the maximum deviation was 6.6113 . The average relative error was $1.9409 \%$, and the maximum relative error was $14.8785 \%$. For $P_{f}=10^{-3}$ the figures were $0.1655,2.4196$, $1.5368 \%$ and $11.8329 \%$, and for $P_{f}=10^{-2}$ : $0.0523,0.6190,1.0345 \%$ and $7.7258 \%$. To summarize, it's safe to state that the common mistakes are less than a $2 \%$ and the maximum mistakes less than a $15 \%$.

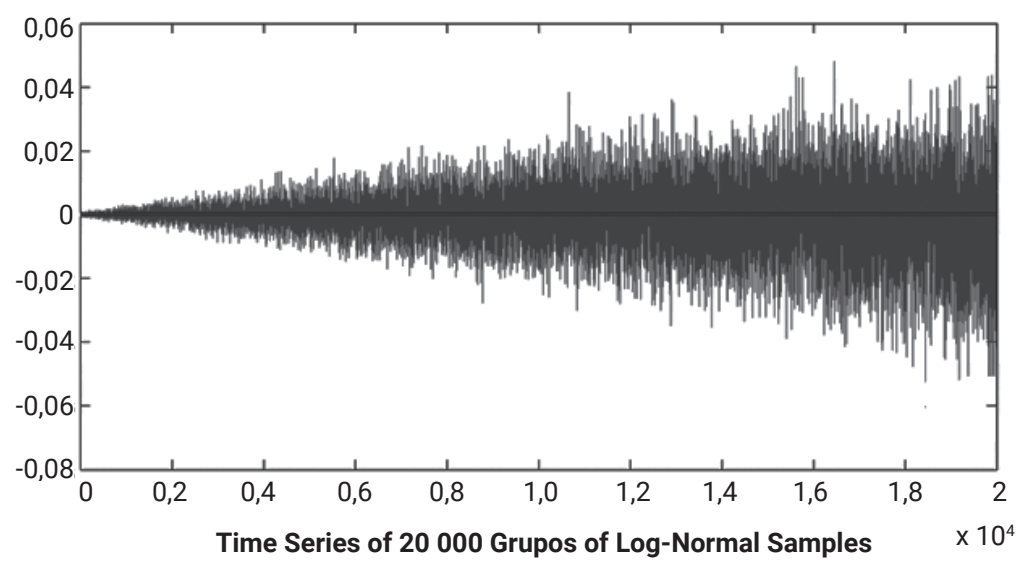

Fig. 6 Magnitude of the errors committed in the estimation of the Log-Normal shape parameter $(\sigma)$ Source: The authors.

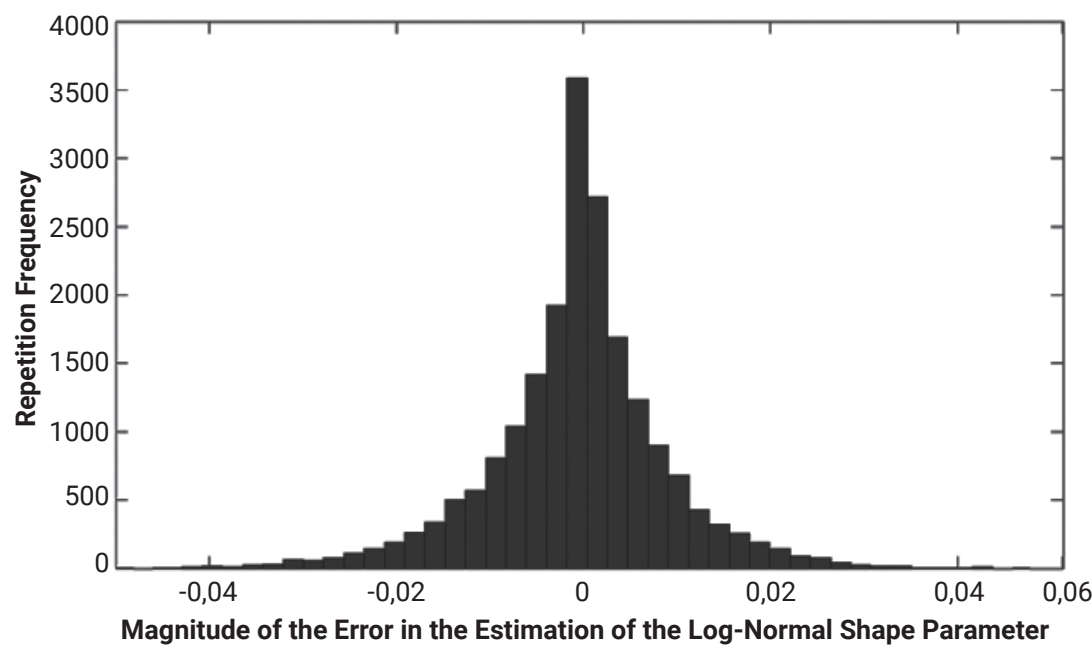

Fig. 7 Histogram of the error in the estimation of the Log-Normal shape parameter $(\sigma)$ Source: The authors. 


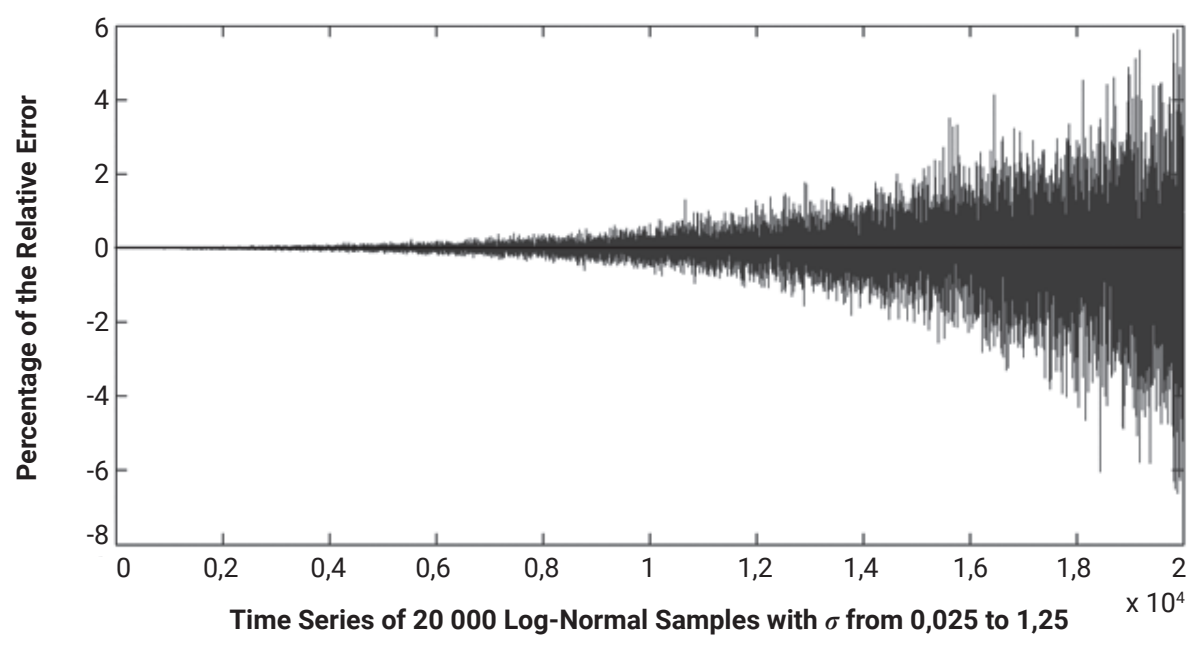

Fig. 8 Absolute error in the selection of the CA-CFAR adjustment factor (T) Source: The authors.

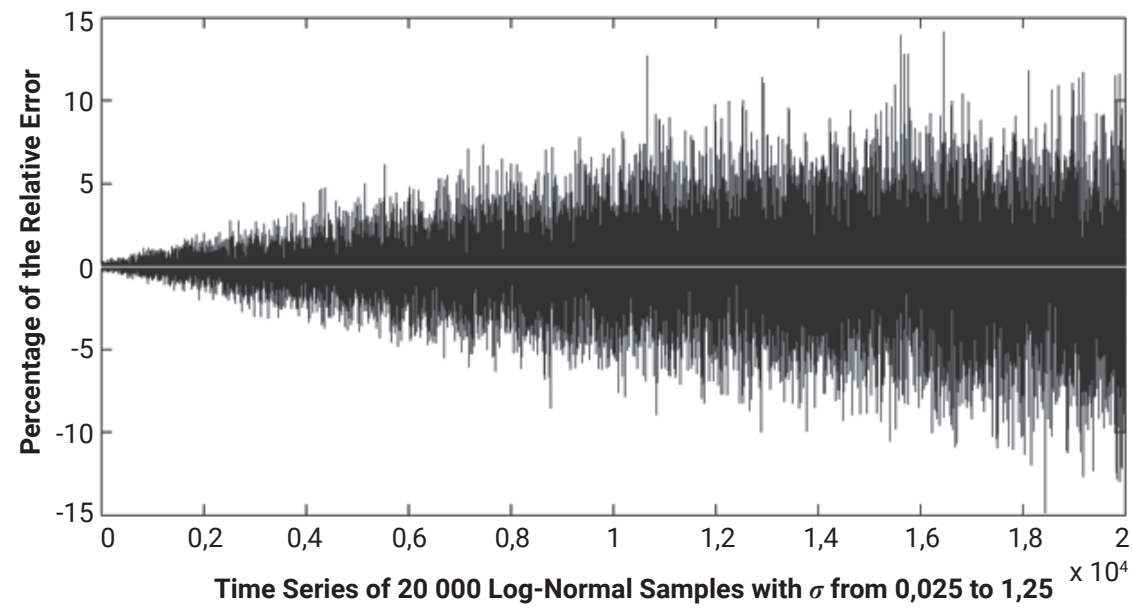

Fig. 9 Relative error in the selection of the CA-CFAR adjustment factor (T) Source: The authors.

\subsection{Deviation in the false alarm probability}

The objective of the new LN-MoM-CA-CFAR is to operate with a minimum deviation of the $P_{f}$ from the design value. The previous tests characterized the deviation of the internal components of the solution. The last test, whose results are shown ahead, used the internal partial results to estimate the overall deviation of the $P_{f}$.

Fig. 10 summarizes the experiments conducted with Set C. Results are plotted for $P_{f}$ $=10^{-2}, P_{f}=10^{-3}$ and $P_{f}=10^{-4}$. Each bin describes the $P_{f}$ deviation experienced after processing 1000 groups of 3000 samples each. The bins to the left in each figure represent 
the lowest values of $\sigma$. Consequently, the bins to the right display a higher magnitude because they represent situations where the detector deals with clutter having a very heavy tailed distribution.

For $P_{f}=10^{-4}$, the average deviation of the operational false alarm probability was
$8.5935 \cdot 10^{-6}$, which represented an $8.5935 \%$ deviation from the design $P_{f}$. For $P_{f}=10^{-3}$, the average departure was $7.27 \cdot 10^{-6}$, for a $0.7270 \%$. Lastly, the average deviation was $2.884 \cdot 10^{-5}$ for $P_{f}=10^{-2}$, which represents a $0.2884 \%$ deviation.
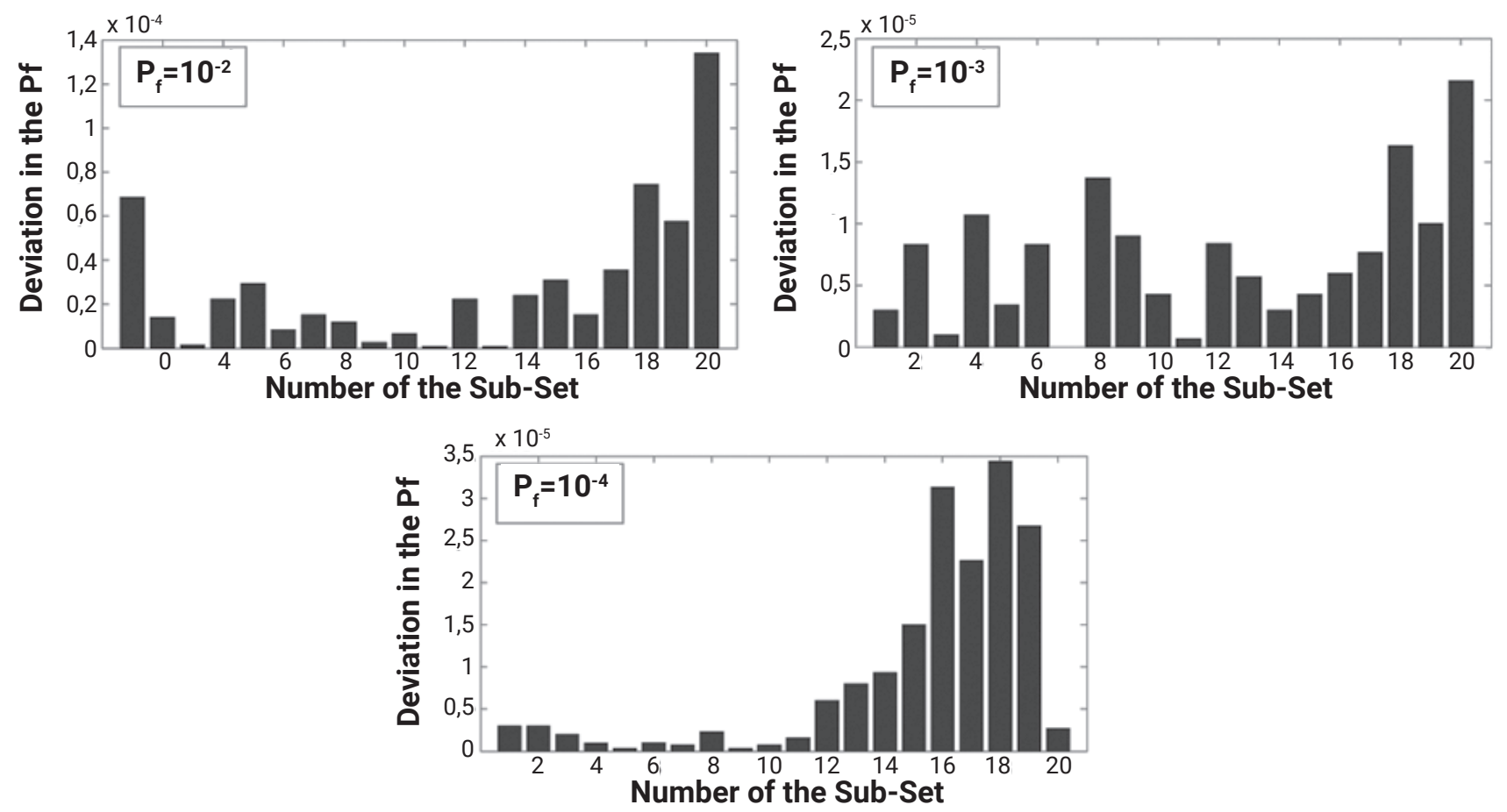

Fig. 10 Deviation of the False Alarm Probability of sub-sets containing 1000 groups of 3000 samples

Source: The authors.

\subsection{Comparing results}

The deviation obtained by the LN-MoM-CACFAR processor from the design $P_{f}$ is very low. This can be appreciated if the results are compared with a similar implementation. In [34] the accuracy of the MoM for the Weibull distribution was measured, and an improved scheme was proposed using neural networks. The average deviations achieved were $4,56 \%, 14,92 \%$ and $34,52 \%$ for the $P_{f}$ s of $10^{-2}$, $10^{-3}$ y $10^{-4}$ respectively. Note that these values are much worse than those achieved for the Log-Normal case $(0,2884 \%, 0,7270 \%$, and $8,5935 \%$ ). 
Additionally, a neuronal solution similar to that proposed in [34] was developed by the authors, but no satisfactory results were found. Fig. 11 shows how the variation of the number of neurons in the hidden layer, main design criteria used in [34], fails to provoke an error inferior to the one exhibited by the MoM in the estimation of $\sigma$.

Additional simulations were conducted with a CA-CFAR with a scale factor fixed to the average clutter behavior (average $\sigma$ ), and it was observed that the processor experienced deviations superior to $200 \%$ even when the whole interval of possible values of $\sigma$ was not swept. The authors could not find any other similar system in the related literature dealing with the problem of correcting the adjustment factor for guaranteeing a reduced deviation from the design false alarm probability. So, they consider that the current proposal is innovative in its field since it solves a problem often ignored by new CFAR schemes, which are usually concentrated of modifying the method for estimating the average of the background.

Although the presented method is designed for the Log-Normal distribution, the applied algorithm may be extended to other clutter related models. The MoM achieves a very accurate estimation of the shape parameter for the Log-Normal case. However, other distribution may require searching other methods.

\section{CONCLUSIONS AND FUTURE RESEARCH}

The new LN-MoM-CA-CFAR radar detector, capable of maintaining a reduced deviation from the design false alarm probability even when facing statistically fluctuating Log-Normal clutter, was presented. The stability of the system is excellent: it's able to operate with a deviation of only $0,2884 \%$ for $P_{f}=10^{-2}$.

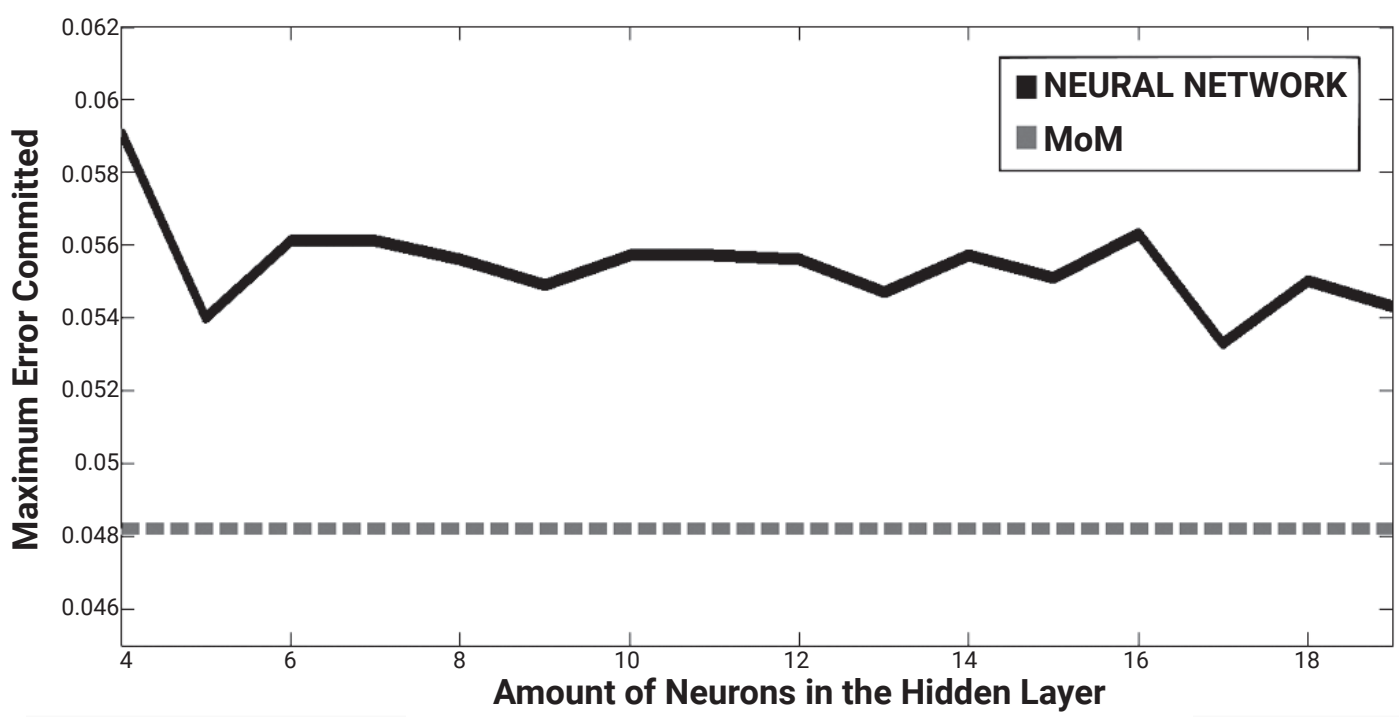

Fig. 11 Comparison between the maximun error of the neural and MoM alternatives Source: The authors. 
The solution solves a problem of proven influence on the performance of radar processors and often ignored in most CFAR proposals.

The proposed detector computes the mean and the variance of the clutter using 3000 samples. Then, it uses this two moments for the estimation of the Log-Normal shape parameter through the method of moments. Finally, a transformation is applied to the computation of the CA-CFAR scale factor. The transformation block constitutes the innovative block of the scheme, and it was completed with the results of Monte Carlo simulations performed over 100 million Log-Normal computer generated samples.

The authors will focus next on developing similar solutions for the popular Compound Gaussian and Pareto statistical distributions that have been related to radar investigations for land and sea clutter. It is also interesting the adaptation of the scheme to the OS-CFAR (Ordered Statistics CFAR) alternative, which would begin to develop a methodology to design CFAR detectors adapted to changes in the clutter.

\section{ACKNOWLEDGMENT}

The authors thank the help offered by Ph.D. Nelson Chávez Ferry regarding the theoretical radar fundamentals and the general conception of the project.

\section{REFERENCES}

[1] M. A. Richards, J. A. Scheer and W. A. Holm. Principles of Modern Radar Vol
I Basic Principles. New Jersey, United States: Scitech Publishing, 2010.

[2] W. L. Melvin and J. A. Scheer. Principles of Modern Radar, Vol III Radar Applications. New Jersey, United States: Scitech Publishing, 2014.

[3] K. Ward, R.Tough and S.Watts. Sea Clutter Scattering, the $K$ Distribution and Radar Performance, (2nd. Ed.). London, United Kingdom: The Institution of Engineering and Technology, 2013. DOI: https://doi.org/10.1049/pbra025e

[4] M. I. Skolnik, Radar Handbook (3er. Ed.). New York, United States: McGraw-Hill, 2008.

[5] H. Meikle. Modern Radar Systems (2nd. Ed.). Boston, United States: Artech House, 2008.

[6] H. Rohling, "Radar CFAR Thresholding in Clutter and Multiple Target Situations," IEEE Transactions on Aerospace and Electronic Systems, vol. 19, no. 4, pp. 608-621, 1983. DOI: https://doi. org/10.1109/TAES.1983.309350

[7] Y. Qin and H. Gong, "A New CFAR Detector based on Automatic Censoring Cell Averaging and Cell Averaging," TELKOMNIKA, vol. 11, no. 6, pp. 3298-3303, 2013. DOI: https://doi. org/10.11591/telkomnika.v11i6.2686

[8] A. Kumar Yadav and L. Kant, "Moving Target Detection using VI-CFAR Algorithm on MATLAB Platform," International Journal of Advanced Research in Computer Science and Software Engineering, vol. 3, no. 12, pp. 915-918, 2013. 
[9] M. B. El Mashade, "Performance Analysis of the Modified Versions of CFAR Detectors in Multiple-Target and Nonuniform Clutter," Radioelectronics and Communications Systems, vol. 56, no. 8, pp. 385-401, 2013. DOI: https://doi. org/10.3103/S0735272713080013

[10] S. W. Shin and Y. K. Seo, "Modified Variability-Index CFAR Detection Robust to Heterogeneous Environment," in Int. Conf. on Systems and Electronic Engineering, Phuket, Thailand, 2012, pp. 6-12.

[11] J. Z. Yim, C. R. Chou and W. K. Wong, "A Study of the Statistics of Sea Clutter in the Northern Coast of Taiwan," in Proceedings of the Seventeenth Int. Offshore and Polar Engineering Conf., Lisbon, Portugal, 2007, pp. 1-6.

[12] Q. Ping, "Analysis of Ocean Clutter for Wide-Band Radar Based on Real Data," in International Conference on Innovative Computing and Cloud Computing, Wuhan, China, 2011, pp. 121-124. DOI: https://doi. org/10.1145/2071639.2071669

[13] Z. Chen, X. Liu and Z. Wu, "The Analysis of Sea Clutter Statistics Characteristics Based on the Observed Sea Clutter of Ku-Band Radar," in IEEE Proceedings of the Int. Symp. on Antennas \& Propagation, 2013, pp. 1183-1186.

[14] A. Mezache, F. Soltani and M. Sahed, "A Model for Non Rayleigh Sea Clutter Amplitudes using Compound Inverse Gaussian," in IEEE Radar Conference (RadarCon13), 2013, Apr. 29-May 3. DOI: https:// doi.org/10.1109/RADAR.2013.6585989
[15] J. R. Machado Fernández and J. C. Bacallao Vidal, "MATE-CFAR: Ambiente de Pruebas para Detectores CFAR en MATLAB,"Telem@tica, vol. 13, no. 3, pp. 86-98, 2014.

[16] S. Sayama and S. Ishii, "Suppression of Log-Normal Distributed Weather Clutter Observed by an S-Band Radar," Wireless Engineering and Technology, vol. 4, no. 3, pp. 125-133, 2013. DOI: https:// doi.org/10.4236/wet.2013.43019

[17] Y. Dong. "Distribution of X-Band High Resolution and High Grazing Angle Sea Clutter," Technical Report DSTO-RR-0316, 2006.

[18] J. R. Machado Fernández and J. C. Bacallao Vidal, "Improved Shape Parameter Estimation in K Clutter with Neural Networks and Deep Learning," International Journal of Interactive Multimedia and Artificial Intelligence, vol. 3, no. 7, pp. 96-103, 2016. DOI: https://doi. org/10.9781/ijimai.2016.3714

[19] J. W. McLeod, "An Investigation of the CDF-Based Method of Moments," Thesis of Master, Department of Civil Engineering, University of Toronto, Toronto, 1998.

[20] J. R. Machado Fernández, "Estimation of the Relation between Weibull Distributed Sea clutter and the CA-CFAR Scale Factor," Journal of Tropical Engineering, vol. 25, no. 2, pp. 19-28, 2015.

[21] J. R. Machado Fernández and J. C. Bacallao Vidal, "Optimal Selection of the CA-CFAR Adjustment Factor for $\mathrm{K}$ Power Sea Clutter with Statistical Variations," Revista Ciencia e Ingeniería Neo- 
granadina, vol. 27, no. 1, pp. 61-76, 2017. DOI: https://doi.org/10.18359/rcin.1714

[22] S. Sayama and S. Ishii, S. "Amplitude Statistics of Sea Clutter by MDL Principle," IEEJ Transactions Fundamentals and Materials, vol. 132, no. 10, pp. 886-892, 2012. DOI: https://doi. org/10.1541/ieejfms.132.886

[23] K. Rajalakshmi Menon, N. Balakrishnan and M. Janakiraman, "Characterization of Fluctuation Statistics of Radar Clutter for Indian Terrain," IEEE Transactions on Geoscience and Remote Sensing, vol. 33, no. 2, pp. 317324, 1995.

[24] S. Sayama and S. Ishii, "Amplitude Statistics of Ground Clutter from Town and Hill Observed by S-band Radar," IEEJ Transactions Fundamentals and Materials, vol. 131, no. 11, pp. 916-923, 2011. DOI: https://doi.org/10.1541/ ieejfms.131.916

[25] Y. Dong. Clutter Spatial Distribution and New Approaches of Parameter Estimation for Weibull and K-Distributions. Edingburgh, South Australia, Australia: DSTO Systems Sciences Laboratory, 2004.

[26] W. Stehwien, "Statistical and Correlation Properties of High Resolution X-band Sea Clutter," in Proceedings of 1994 IEEE National Radar Conference, pp. 46-51, 1994. DOI: https://doi. org/10.1109/NRC.1994.328096

[27] A. Farina, F. Gini and M. V. Greco, "High Resolution Sea Clutter Data: Statistical Analysis of Recorded Live Data," IEE Proceedings on Radar, Sonar and $\mathrm{Na}$ vigation, vol. 144 , no. 3 , pp. 121-130,
1997. DOI: https://doi.org/10.1049/ ip-rsn:19971107

[28] S. Ishii, S. Sayama and K. Mizutani, "Effect of Changes in Sea-Surface State on Statistical Characteristics of Sea Clutter with X-band Radar," Wireless Engineering and Technology, vol. 2, no. 3, pp. 175-183. DOI: https://doi. org/10.4236/wet.2011.23025

[29] N. Weiping, Y. Weidong and B. Hui, "Statistical Analysis of High Resolution TerraSAR-X Images for Ground Targets Detection," in Seventh International Conference on Image and Graphics, Qingdao, China, Jul. 26-28, 2013, pp. 343-347. DOI: https://doi.org/10.1109/ICIG.2013.74

[30] J. Jurgens Strydom, "Generic Ground Clutter Simulation for Radar Testing and Evaluation," Thesis of Master, Radar Remote Sensing Group, Department of Electrical Engineering, University of Cape Town, Cape Town, 2012.

[31] K. Ward, R. Tough and S. Watts. Sea Clutter. Scattering, the $K$ distribution and Radar Performance. London, United Kingdom: The Institution of Engineering and Technology, 2006.

[32] M. Greco, F. Bordoni and F. Gini, "X-Band Sea-Clutter nonstationarity: Influence of Long Waves," IEEE Journal of Oceanic Engineering, vol. 29, no. 2, pp. 269-283, 2004. DOI: https://doi. org/10.1109/JOE.2004.828548

[33] S. Sayama and M. Sekine, "Weibull, Log-Weibull and K-Distributed Ground Clutter Modelling Analyzed by AIC," IEEE Transactions on Aerospace and Electronic Systems, vol. 37, no. 3, pp. 
1108-1113, 2001. DOI: https://doi. org/10.1109/7.953262

[34] J. R. Machado Fernández and J. C. Bacallao Vidal, "Procesador de Promediación con Corrección Adaptativa del Factor de Ajuste Mediante Redes Neu- ronales Artificiales," in VIIII Congreso Internacional de Telemática y Telecomunicaciones, Convención Científica de Ingeniería y Arquitectura '16, Palacio de las Convenciones, (La Habana, Cuba), Oct. 21-25, 2016, pp. 237-246. 